\title{
Spin-anisotropic magnetic impurity in a Fermi gas: poor man's scaling equation integration
}

\author{
Eugene Kogan, ${ }^{1,2, \text { 田 Kazuto Noda, }}{ }^{2}$ and Seiji Yunoki ${ }^{2}, 3,4,5$ \\ ${ }^{1}$ Jack and Pearl Resnick Institute, Department of Physics, Bar-Ilan University, Ramat-Gan 52900, Israel \\ ${ }^{2}$ Computational Quantum Matter Research Team, \\ RIKEN Center for Emergent Matter Science (CEMS), Wako, Saitama 351-0198, Japan \\ ${ }^{3}$ Computational Condensed Matter Physics Laboratory, RIKEN, Wako, Saitama 351-0198, Japan \\ ${ }^{4}$ Interdisciplinary Theoretical Science (iTHES) Research Group, RIKEN, Wako, Saitama 351-0198, Japan \\ ${ }^{5}$ Computational Materials Science Research Team, \\ RIKEN Advanced Institute for Computational Science (AICS), Kobe, Hyogo 650-0047, Japan
}

(Dated: September 2, 2018)

\begin{abstract}
We consider a single magnetic impurity described by the spin-anisotropic s-d(f) exchange (Kondo) model and formulate scaling equation for the spin-anisotropic model when the density of states (DOS) of electrons is a power law function of energy (measured relative to the Fermi energy). We solve this equation containing terms up to the second order in coupling constants in terms of elliptic functions. From the obtained solution we find the phases corresponding to the infinite isotropic antiferromagnetic Heisenberg exchange, to the impurity spin decoupled from the electron environment (only for the pseudogap DOS), and to the infinite Ising exchange (only for the diverging DOS). We analyze the critical surfaces, corresponding to the finite isotropic antiferromagnetic Heisenberg exchange for the pseudogap DOS.
\end{abstract}

PACS numbers: 75.50.Mm, 72.15.Qm, 03.75.Mn

\section{INTRODUCTION}

Kondo model, describing a magnetic moment embedded in a system of non-interacting fermions continues to attract attention of both theorists and experimentalists for more than half a century. In spite of the model seeming simplicity, it is well known that the model is as far from being simple, as one can get, especially when additional complicated factors, like spin-anisotropy or nonflat behaviour of the electrons density of states (DOS) are to be taken into account. A very insightful approach to this model is based on scaling equation pioneered by Anderson 1,2. As far as spin-anisotropic model is concerned, to the best of our knowledge only the case of the $X X Z$ model (see below) with the flat DOS was analysed in a detailed way ${ }^{2}$.

A pseudogap Kondo model, with a power-law DOS $\rho(\epsilon) \sim|\epsilon|^{r}$, which has recently attracted a lot of attention ${ }^{3}-\underline{6}$ (for review, see Ref. 7). In particular, graphene, where the Kondo effect was observed recently ${ }^{8}$, is considered as a typical realization of this model. More generally, one is interested in Kondo problem for spin coupled to electrons with the pseudogap or diverging $\operatorname{DOS}^{9}-15$.

A model of a Kondo-like impurity interacting spinisotropically with a band of fermions for which the DOS is zero or small near the Fermi energy was considered in Ref. 16. There renormalization-group arguments were used to demonstrate that this model has a nontrivial zero-temperature phase transition at a finite coupling constant, in contrast to the zero-coupling-constant transition of the Kondo model with constant DOS. Numerical and perturbative renormalization study on Kondo and Anderson models have provided a comprehensive understanding of phase diagrams and thermodynamic properties $7,17-22$. However, a spin-anisotropic pseudogap Kondo model to our best knowledge was never considered.

The rest of the paper is constructed as follows. We formulate in Section II poor man's scaling equation for the spin-anisotropic model with the power law DOS. In Section III solutions of scaling equations satisfying $J_{x}=J_{y}$ condition, referred to as $X X Z$ model, are presented as a preparation for the Section IV] where we integrate the general scaling equation for the spin-anisotropic model. Some important mathematical details and geometric interpretation of the solution are relegated to the Appendix.

\section{POOR MAN'S SCALING FOR THE SPIN-ANISOTROPIC MODEL}

\section{A. Hamiltonian and scaling equation}

Poor man's scaling is the renormalization idea applied to the model of a single magnetic impurity in the Fermi sea of itinerant electrons. The Hamiltonian of the model can be written as

$$
H=H_{0}+V=\sum_{\mathbf{k} \alpha} \epsilon_{\mathbf{k}} c_{\mathbf{k} \alpha}^{\dagger} c_{\mathbf{k} \alpha}+V,
$$

where $c_{\mathbf{k} \alpha}^{\dagger}$ and $c_{\mathbf{k} \alpha}$ are electron creation and annihilation operators, $\epsilon_{\mathbf{k}}$ is the energy of itinerant electron with wave vector $\mathbf{k}$ and spin $\alpha$, and the operator $V$ describes interaction between the electrons and the impurity.

To formulate the renormalization procedure we need three objects: the Hamiltonian of the system $H$, the 
Hilbert space $\mathcal{H}$ (which is the product of the band of itinerant electrons of width $D$ and of the Hilbert space where the impurity lives), and the $T$ matrix, given by the series

$$
T=V+V G_{0} V+V G_{0} V G_{0} V+\ldots,
$$

where $G_{0}=\left(E+i 0-H_{0}\right)^{-1}$.

Suppose we are interested only in the matrix elements of the $T$ matrix between the electron states at a distance from the Fermi energy much less than the band width. Can we ignore the band edges? The answer is "No", because of virtual transitions of the electrons to the band edges, and the virtual transitions is all what quantum mechanics is about. Can we take into account all virtual transitions using perturbative expansion (2)? The answer is again "No", because the series diverges due to transitions to the stats close to the Fermi energy.

The brilliant idea of Anderson was that we can take into account virtual transition to the band edges perturbatively, that is by taking into account only a few first terms in Eq. (2), thus reducing the band width $D$ of the itinerant electrons and calculating the renormalization of the Hamiltonian due to the elimination of the above mentioned virtual transitions. Thus we reduce the Hilbert space $\mathcal{H}$ and renormalize the Hamiltonian $H$ accordingly, to keep the $T$ matrix constant. And we can repeat this procedure again and again.

Now let us consider Kondo model. We find it appropriate to write down the exchange part of the Hamiltonian in explicitly rotation invariant form

$$
V=H_{\mathrm{ex}}=\sum_{i j} J_{i j} S^{i} s^{j}(0),
$$

where $\vec{S}$ is the (siting at $\mathbf{r}=0$ ) impurity spin operator (spin is one half), $\vec{s}(0)=\frac{1}{2} \sum_{\mathbf{k k}^{\prime} \alpha \beta} c_{\mathbf{k}^{\prime} \alpha}^{\dagger} \vec{\sigma}_{\alpha \beta} c_{\mathbf{k} \beta}$, $\left(\sigma^{x}, \sigma^{y}, \sigma^{z}\right.$ are Pauli matrices) is the itinerant electrons spin operator, and $J_{i j}$ are the anisotropic exchange coupling constants.

Let us consider first the traditional case of flat DOS. Poor Man's scaling consists in reducing the band width $D$ of the itinerant electrons and calculating perturbatively the renormalized interactions due to the elimination of the virtual excitations to the band edges. For the isotropic model

$$
H_{\mathrm{ex}}=J \vec{S} \cdot \vec{s}(0)
$$

scaling equation in the lowest order is 1,2

$$
\frac{d J}{d \ln \Lambda}=-2 \rho J^{2},
$$

where $\rho$ is DOS.

Consider generalization of Hamiltonian (3):

$$
H_{\mathrm{ex}}=\sum_{i j=1}^{N^{2}-1} J_{i j} S^{i} T^{j}
$$

where $S^{i}$ and $T^{j}$ are traceless generators of the group $S U(N)$.

Quadratic term in the scaling equation appears due to elimination of virtual transition of electron to the band edges in the lowest order of perturbation theory. Hence, in the process of renormalization of the anisotropic Hamiltonian there appears the factor

$$
\begin{aligned}
& J_{i j} S^{i} T^{j} J_{k l} S^{k} T^{l} \\
& =\frac{1}{4} J_{i j} J_{k l}\left(\left\{S^{i}, S^{k}\right\}\left\{T^{j}, T^{l}\right\}+\left[S^{i}, S^{k}\right]\left[T^{j}, T^{l}\right]\right)
\end{aligned}
$$

(in Eqs. (77)- (10) we accept the summation convention: in all equations summation from 1 to $N^{2}-1$ with respect to every repeated index is implied).

Taking into account that

$$
\begin{aligned}
\left\{S_{i}, S_{k}\right\} & =\frac{\delta_{i k}}{N}+d_{i k n} S_{n} \\
{\left[S_{i}, S_{k}\right] } & =i f_{i k n} S_{n},
\end{aligned}
$$

where $N \times N$ unit matrix is suppressed, the $d$-coefficients are symmetric in all indices, and $f$ are structure constants, we obtain

$$
\begin{aligned}
& 4 J_{i j} S^{i} T^{j} J_{k l} S^{k} T^{l}=\frac{1}{N^{2}} J_{i j} J_{i j} \\
& +\frac{1}{N} J_{i j} J_{j k} d_{i k m}\left(S^{m}+T^{m}\right) \\
& +J_{i j} J_{k l}\left(d_{i k m} d_{j l n}-f_{i k m} f_{j l n}\right) S^{m} T^{n} .
\end{aligned}
$$

Thus for the $S U(2)$ Kondo model one obtains 23,24

$$
\frac{d J_{m n}}{d \ln \Lambda}=-\rho J_{i j} J_{k l} \epsilon_{i k m} \epsilon_{j l n},
$$

where $\epsilon$ is Levi-Civita symbol.

The microscopic tensor $J_{i k}^{(m)}$ can always be reduced to principal axes by rotation of the coordinate system, and it keeps it's diagonal form in the process of renormalization, as we see from Eq. (10). So we can write down the exchange Hamiltonian as

$$
H_{\mathrm{ex}}=\sum_{i} J_{i} S^{i} s^{i}(0),
$$

and the scaling equation as

$$
\begin{aligned}
& \frac{d J_{x}}{d \ln \Lambda}=-2 \rho J_{y} J_{z} \\
& \frac{d J_{y}}{d \ln \Lambda}=-2 \rho J_{x} J_{z} \\
& \frac{d J_{z}}{d \ln \Lambda}=-2 \rho J_{x} J_{y},
\end{aligned}
$$

and sweep under the carpet the question of stability of the diagonal solution by adding to the poor man's scaling a possible rotation of coordinate system at each step. ${ }^{1}$

\footnotetext{
1 Actually, the Hamiltonian (3) (or (11)) is more meaningful for the theory of two level systems, $S^{i}$ and $s^{i}$ being pseudospin operators $^{23}$, than for the orthodoxal Kondo model 25 .
} 
Now let us return to the case when the electron dispersion law determines the power law dependence of the DOS upon the energy

$$
\rho(\epsilon)=C|\epsilon|^{r}, \text { if }|\epsilon|<D,
$$

where $r$ can be either positive or negative $(r>-1)^{14}$. For the DOS we consider, in distinction from the standard renormalization procedure ${ }^{2}$, one has additionally to rescale the unit of length ${ }^{16}$. Thus for the isotropic model (4) scalar scaling equation is ${ }^{2}$

$$
\frac{d J}{d \ln \Lambda}=r J-2 G J^{2}
$$

where $G=C D^{r}$, and $\Lambda=D^{\prime} / D ; D^{\prime}$ is the actual width of the itinerant electrons band after the exclusion of the virtual excitations to the edges, was obtained and studied previously $\stackrel{16}{ }$.

Combining spin-anisotropy and power law DOS we should add linear terms to the RHS of Eq. (12) (with zero $N$ ) to obtain

$$
\begin{aligned}
& \frac{d J_{x}}{d \ln \Lambda}=r J_{x}-2 G J_{y} J_{z} \\
& \frac{d J_{y}}{d \ln \Lambda}=r J_{y}-2 G J_{x} J_{z} \\
& \frac{d J_{z}}{d \ln \Lambda}=r J_{z}-2 G J_{x} J_{y} .
\end{aligned}
$$

\section{B. Symmetries and fixed points of the scaling equation}

Equation (10) is symmetric with respect to all space rotations (the group $K^{26}$ ), from which only the symmetry with respect to permutation of the indices $x, y, z$ is left for Eq. (15). Additionally, both equations are symmetric with respect to space inversion accompanied by the inversion of the direction of flow and change of sign of $r$. So further on we consider explicitly only the case $r>0$ (and present the results for negative $r$ in some cases).

We introduce $\lambda=\Lambda^{r}$ and, unless $G$ appear explicitly in the equation, measure $J$ in units of $r / 2 G$. So Eq. (15) becomes

$$
\begin{aligned}
& \lambda \frac{d J_{x}}{d \lambda}=J_{x}-J_{y} J_{z} \\
& \lambda \frac{d J_{y}}{d \lambda}=J_{y}-J_{x} J_{z} \\
& \lambda \frac{d J_{z}}{d \lambda}=J_{z}-J_{x} J_{y} .
\end{aligned}
$$

\footnotetext{
${ }^{2}$ It is known that physics of the problem can change at $r=$ $1 / 2^{7,16}$, and thus the range of validity of scaling equations [15] is an open problem. However, we do not discuss here this problem and admit the limitation of our approach: the value of $r$ determines just the scale of the problem (see below).
}

When we look for the flow lines of Eq. (16), the parameter $\lambda \in(0,+\infty)$ (and decreases along a flow line). When we consider the physical problem, the parameter $\lambda \in(0,+1]$, and Eq. (16) becomes the initial (final) value problem with

$$
J_{n}(1)=2 G J_{n}^{(m)} / r .
$$

Equation (16) has a trivial fixed point

$$
J_{x}^{*}=J_{y}^{*}=J_{z}^{*}=0,
$$

corresponding to the impurity spin decoupled from the electron environment, and four non-trivial ones

$$
\left|J_{x}^{*}\right|=\left|J_{y}^{*}\right|=\left|J_{z}^{*}\right|=1 ; \quad J_{x} J_{y} J_{z}=1,
$$

corresponding to finite isotropic antiferromagnetic Heisenberg exchange.

Apart from the finite fixed points given by Eqs. (18) and (19), Eq. (16) has infinite fixed points (more precisely, rays starting at the origin and going to the infinity, which serve as attractors for the flow lines). However, it will be more convenient to discuss these attractors later (in the Subsection IVA).

It is obvious that the trivial fixed point is stable. (We remind that we consider here only the case of positive $r$.) To analyze stability of the non-trivial fixed points, we write $J_{n}=J_{n}^{*}+\delta J_{n}$ and linearize Eq. (16) with respect to deviations from the fixed point $\delta J_{n}$. Thus we obtain

$$
\frac{d \delta J_{n}}{d \lambda}=\sum_{m} T_{n m} \delta J_{m}
$$

where the eigenvalues of matrix $T$ for any fixed point are -1 and doubly degenerate 2 . Hence all the nontrivial fixed points are semi-stable and, hence, are critical points.

Taking the second step we introduce $\widetilde{J}_{n}=J_{n} / \lambda$, and Eq. (15) takes the form

$$
\begin{aligned}
& \frac{d \widetilde{J}_{x}}{d \lambda}=-\widetilde{J}_{y} \widetilde{J}_{z} \\
& \frac{d \widetilde{J}_{y}}{d \lambda}=-\widetilde{J}_{x} \widetilde{J}_{z} \\
& \frac{d \widetilde{J}_{z}}{d \lambda}=-\widetilde{J}_{x} \widetilde{J}_{y} .
\end{aligned}
$$

\section{INTEGRATION OF SCALING EQUATIONS FOR THE $X X Z$ MODEL}

\section{A. What we can learn from isotropic model}

We start our analysis from the simple case of isotropic model $\left(J_{x}=J_{y}=J_{z}=J\right)$, though it was analyzed before. However we prefer to reproduce the analysis, because this way we understand the pattern, which will 
repeat itself throughout the paper. Eq. (21) in this case can be solved immediately

$$
\widetilde{J}=\frac{1}{\lambda+\psi} \Longrightarrow J=\frac{\lambda}{\lambda+\psi} .
$$

From the point of view of a mathematician, $\psi$ is just the constant of integration. From the point of view of a physicist $\psi$ for the particular problem has the particular value, connected with microscopic parameters by Eq. (17). For $\psi>0$, when $\lambda$ decreases to zero, $J(\lambda)$ converges to the trivial fixed point, which means that the spin is decoupled from the environment (when energy goes to zero). The value of $\psi=0$ means a critical point, that is energy independent interaction of the spin with the environment. And finally, if $-1<\psi<0$, then $J(\lambda)$ has a relevant pole at some finite value of $\lambda$, corresponding to finite value of $D^{\prime}$. (This pole is similar to Landau pole ${ }^{27}$.)

Formally, this pole just means that the perturbation theory (and scaling equation (15) is a clever but still perturbation theory) breaks down. On the other hand, as it is known since long ago, the value of $D^{\prime}$ mentioned above provides an estimate of Kondo temperature $T_{K}$

$$
T_{K} \sim D^{\prime}=D(-\psi)^{1 / r}
$$

So the parameter $\psi$ has a clear physical meaning.

Substituting Eq. (22) into Eq. (17) one obtains 13

$$
T_{K} \sim D\left(1-\frac{r}{2 G J^{(m)}}\right)^{1 / r},
$$

provided

$$
0<\frac{r}{2 G J^{(m)}}<1
$$

Note that if we take the limit $r \rightarrow 0$ in Eq. (24), we obtain

$$
T_{K} \sim D e^{-1 / 2 G J^{(m)}}
$$

\section{B. The $X X Z$ model}

Now let us go to the $X X Z$ model $\left(J_{x}=J_{y}\right)$. Eq. (21) in this case takes the form

$$
\begin{aligned}
& \frac{d \widetilde{J}_{x}}{d \lambda}=-\widetilde{J}_{z} \widetilde{J}_{x} \\
& \frac{d \widetilde{J}_{z}}{d \lambda}=-\widetilde{J}_{x}^{2} .
\end{aligned}
$$

We immediately obtain the first integral of Eq. (27)

$$
\widetilde{J}_{x}^{2}-\widetilde{J}_{z}^{2}= \pm A^{2}
$$

Substituting into Eq. (27) and integrating we get

$$
\begin{aligned}
& J_{x}= \pm A \lambda \cdot \csc (\mathrm{h})(A \lambda+\psi) \\
& J_{z}=A \lambda \cdot \cot (\mathrm{h})(A \lambda+\psi) .
\end{aligned}
$$

In Eq. 29) $\cos (\mathrm{h})$ stands for either trigonometric or hyperbolic cosine, and similar for $\cot (\mathrm{h})$.

Note that presence of two integration constants $(\psi$ and $A$ ) in the solution (29) reflects two symmetries of Eq. (21): with respect to transformation $\lambda \rightarrow \lambda+\psi$ and with respect to transformation $\lambda \rightarrow A \lambda, \widetilde{J} \rightarrow \widetilde{J} / A$. For trigonometric functions $\psi \in(-\pi / 2, \pi / 2]$, for hyperbolic functions $\psi \in(-\infty,+\infty)$.

Together with fixed points, separatrices form the skeleton of a flow diagram. In our case non-trivial separatrices are described by putting $\psi=0$ in Eq. (29). Thus we get four separatrices (ending at the critical points), described by the equations

$$
\frac{\widetilde{J}_{z}}{\left|\widetilde{J}_{x}\right|}=\cos (\mathrm{h})\left(\sqrt{\left|\widetilde{J}_{x}^{2}-\widetilde{J}_{z}^{2}\right|}\right)
$$

(in the case of cos the solution of Eq. (30) should also satisfy $\left.\sqrt{\widetilde{J}_{x}^{2}-\widetilde{J}_{z}^{2}}<\pi\right)$. The asymptotic of the solution of Eq. (30) is

$$
\begin{aligned}
& \widetilde{J}_{x}= \pm\left(\widetilde{J}_{z}-\frac{\pi^{2}}{2 \widetilde{J}_{z}}\right), \quad \widetilde{J}_{z} \ll-1 \\
& \widetilde{J}_{x}= \pm \widetilde{J}_{z} e^{-\widetilde{J}_{z}}, \quad \widetilde{J}_{z} \gg 1 .
\end{aligned}
$$

The trivial separatrices are $\widetilde{J}_{x}=0$ and $\widetilde{J}_{x}= \pm \widetilde{J}_{z}$.

A flow diagram, as described by Eq. (29), is shown in Fig. 1. Because of the symmetry of Eq. (16) it is enough to plot only the upper part of the phase plain $J_{x} \geq 0$. We observe the non-interacting phase, corresponding to the trivial fixed point, the phase of infinite isotropic antiferromagnetic Heisenberg exchange, (both phases corresponding to stable fixed points) We also observe the critical line of finite isotropic antiferromagnetic Heisenberg exchange, ending at the critical point (semi-stable fixed point). This Figure shows an example of asymptotic symmetry ${ }^{28}$. After the renormalization the system becomes isotropic (or trivial) even it was anisotropic microscopically.

The same flow diagram as in Fig. 1, is shown in Fig. 2, but this time the plot includes larger values of $J_{x}, J_{z}$ (or smaller values of $r$ ). The main purpose of this Figure is to illustrate how our results are reduced to those obtained for the constant DOS ${ }^{2}$ when $r \rightarrow 0$. For large $J_{x}, J_{z}$ the linear terms in Eq. (27) can be neglected, and the flow diagram naturally looks like the one from Ref. 2, which consists of hyperbolas. However, when in the process of evolution at least one of $J_{x}, J_{z}$ becomes of order one, the principal deviations from the hyperbolas can be clearly seen. Fig. 2 also illustrates how the fixed line $J_{x}=0$ obtained for $r=0^{2}$ emerges when $r \rightarrow 0$.

Flow diagram for negative $r$, presented on Fig. 3, is just the diagram from Fig. 1, replotted taken into account the symmetry of the problem mentioned in the beginning of the Subsection IB. Because change of sign of $r$ should be accompanied by the inversion of the direction of flow (and space inversion), after this change the 
previously stable fixed points become unstable (and of no physical interest) and vice versa. Thus for negative $r$ we have two phases, corresponding respectively to infinite isotropic antiferromagnetic Heisenberg exchange and infinite Ising exchange. We want to emphasise again, that physical fixed points for negative $r$ correspond to nonphysical fixed points for positive $r$. Notice also that for negative $r$ the critical point is ferromagnetic (and the critical line is totally different from that for positive $r$ ). Thus Fig. 3 shows (depending upon the initial conditions) either the same asymptotic symmetry we had for positive $r$ or dynamical generation of anisotropy.

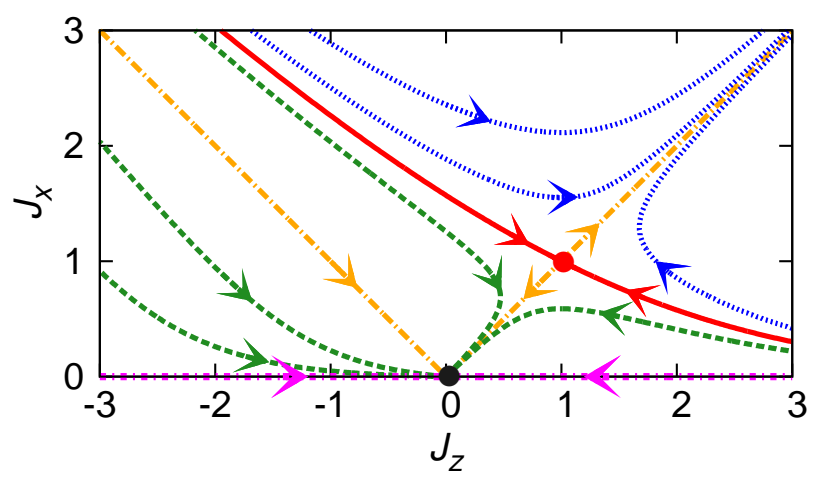

FIG. 1: (color online) Flow diagram as described by Eq. (29). Trivial fixed point is shown by black circle, critical point by red circle, isotropic model by orange dot dashed line, Ising model by violet dot dot dashed line, critical line by red solid line. The non-interacting (infinite isotropic antiferromagnetic Heisenberg exchange) phase is shown by green dashed (blue dotted) lines.

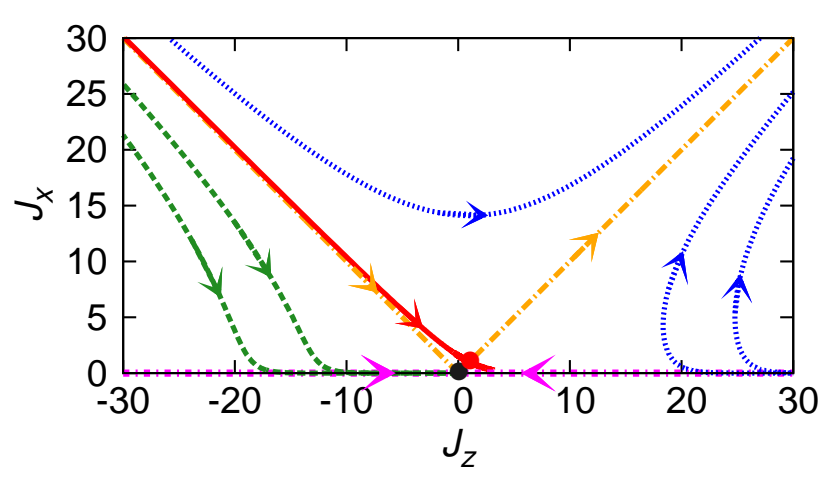

FIG. 2: (color online) Same as Fig. 1, but with a wider plot interval.

As far as Kondo effect is concerned, we can repeat verbatim the two paragraphs following Eq. (22), only $\psi$ in the RHS of Eq. (223) should be divided by $A$. The values of $\psi$ and $A$ are found this time by substituting Eq. (29) into Eq. (17). ${ }^{3}$

\footnotetext{
${ }^{3}$ We recently learned that part of results of the Subsection
}

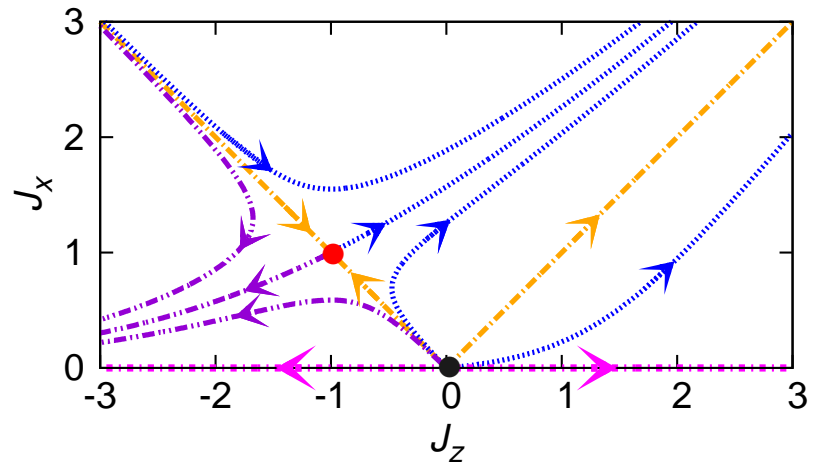

FIG. 3: (color online) Flow diagram for negative $r$. The infinite Ising exchange phase is shown by magenta dot short dashed lines.

\section{INTEGRATION OF SCALING EQUATIONS IN THE GENERAL CASE}

\section{A. Tale of two integrals}

Let us generalize Eq. (21) to

$$
\begin{aligned}
& \frac{d \widetilde{J}_{x}}{d \lambda}=Q \widetilde{J}_{y} \widetilde{J}_{z} \\
& \frac{d \widetilde{J}_{y}}{d \lambda}=R \widetilde{J}_{x} \widetilde{J}_{z} \\
& \frac{d \widetilde{J}_{z}}{d \lambda}=S \widetilde{J}_{x} \widetilde{J}_{y},
\end{aligned}
$$

where $Q, R, S$ are some constants. Equation (32) includes the $X Y Z$ model $(P=Q=R=-1)$, and also other, much more important cases, like Euler top $\underline{30}$.

From Eq. (32) follows

$$
\frac{d}{d \lambda}\left(a \widetilde{J}_{x}^{2}+b \widetilde{J}_{y}^{2}+c \widetilde{J}_{z}^{2}\right)=0,
$$

where $a, b, c$ are arbitrary constants satisfying

$$
a Q+b R+c S=0 .
$$

Hence we immediately get two first integrals

$$
\begin{aligned}
& a_{1} \widetilde{J}_{x}^{2}+b_{1} \widetilde{J}_{y}^{2}+c_{1} \widetilde{J}_{z}^{2}=A_{1} \\
& a_{2} \widetilde{J}_{x}^{2}+b_{2} \widetilde{J}_{y}^{2}+c_{2} \widetilde{J}_{z}^{2}=A_{2},
\end{aligned}
$$

where $\left(a_{1}, b_{1}, c_{1}\right)$ and $\left(a_{2}, b_{2}, c_{2}\right)$ should be linearly independent. Equations (35) seems to contain a lot of constants, but if we consider it (and Eq. (34) as defining straight line in the space with the coordinates $(x, y, z)=$ $\left({\widetilde{J_{x}}}^{2},{\widetilde{J_{y}}}^{2},{\widetilde{J_{z}}}^{2}\right)$, we are motivated to present this equation in the canonical form

$$
\frac{x-x_{0}}{Q}=\frac{y-y_{0}}{R}=\frac{z-z_{0}}{S} .
$$

were independently obtained in Ref. 29 . 
Thus in the space introduced above each flow lines lies on the ray with the direction $(Q, R, S)$.

Returning temporarily to the Kondo problem we solve $(Q=R=S=-1)$, we immediately understand by inspection of Eq. (36) that the attractors of Eq. (16) going to infinity corresponds to isotropic Hamiltonian.

\section{B. General solution}

Two integrals being found, we are left with a single equation for a single variable $\mathcal{P}$, which is naturally to chose according to the equation

$$
\frac{x-x_{0}}{Q}=\frac{y-y_{0}}{R}=\frac{z-z_{0}}{S}=\frac{\mathcal{P}}{Q R S} .
$$

Also, Eq. (35) contains just two constants. To emphasize this fact we put on $x_{0}, y_{0}, z_{0}$ condition

$$
\frac{x_{0}}{Q}+\frac{y_{0}}{R}+\frac{z_{0}}{S}=0 .
$$

Substituting Eq. (37) into Eq. (32) and taking into account Eq. (38) we obtain 31

$$
\left[\frac{d \mathcal{P}(\lambda)}{d \lambda}\right]^{2}=4[\mathcal{P}(\lambda)]^{3}-g_{2} \mathcal{P}(\lambda)-g_{3},
$$

where

$$
\begin{aligned}
& g_{2}=-4\left(R S x_{0}+Q S y_{0}+Q R z_{0}\right) \\
& g_{3}=-4 Q^{2} R^{2} S^{2} x_{0} y_{0} z_{0} .
\end{aligned}
$$

Hence $\mathcal{P}(\lambda)$ is Weierstrass elliptic function $\mathcal{P}\left(\lambda ; \omega_{1}, \omega_{2}\right)^{32}$, and $\omega_{1}, \omega_{2}$ are connected with $g_{2}, g_{3}$ by equation

$$
\begin{aligned}
& g_{2}=60 \sum_{(m, n) \neq(0,0)}\left(m \omega_{1}+n \omega_{2}\right)^{-4} \\
& g_{3}=140 \sum_{(m, n) \neq(0,0)}\left(m \omega_{1}+n \omega_{2}\right)^{-6} .
\end{aligned}
$$

Thus we obtain the solution of Eq. (16) as

$$
\begin{aligned}
J_{x}^{2} & =\lambda^{2}\left[\mathcal{P}\left(\lambda+\psi ; \omega_{1}, \omega_{2}\right) / R S+x_{0}\right] \\
J_{y}^{2} & =\lambda^{2}\left[\mathcal{P}\left(\lambda+\psi ; \omega_{1}, \omega_{2}\right) / Q S+y_{0}\right] \\
J_{z}^{2} & =\lambda^{2}\left[\mathcal{P}\left(\lambda+\psi ; \omega_{1}, \omega_{2}\right) / Q R+z_{0}\right] .
\end{aligned}
$$

The solution represents a two-parameter $\left(\omega_{1}, \omega_{2}\right)$ family of the flow lines, with $x_{0}, y_{0}, z_{0}$ being connected with these two parameters by Eqs. (38), (40), (41).

Alternative (and more convenient) representation of the solution of Eq. (16) through Jacobi elliptic functions is presented in the Appendix. The result for the problem we solve is

$$
\begin{aligned}
& J_{x}= \pm A \lambda \cdot \operatorname{ns}(A \lambda+\psi, k) \\
& J_{y}= \pm A \lambda \cdot \operatorname{cs}(A \lambda+\psi, k) \\
& J_{z}= \pm A \lambda \cdot \operatorname{ds}(A \lambda+\psi, k),
\end{aligned}
$$

where the factors \pm 1 should satisfy condition that their product is equal to +1 , plus the solutions which can be obtained from Eq. (43) by interchanging $J_{x}, J_{y}, J_{z}$.

Equation (43) is the main result of the paper. It represents a two-parameter family of the flow lines. The parameter $\psi \in(-K(k), K(k)]$, where $K$ is the complete elliptic integral of the first kind, and the parameter $k \in[0,1]$, and, as we show in the appendix, has the simple geometric meaning.

The results of the previous Section are the particular case of those obtained in this Section. In fact, because

$$
\left\{\begin{array} { l } 
{ \mathrm { ns } ( \phi , 0 ) = \operatorname { c s c } ( \phi ) } \\
{ \operatorname { c s } ( \phi , 0 ) = \operatorname { c o t } ( \phi ) } \\
{ \operatorname { d s } ( \phi , 0 ) = \operatorname { c s c } ( \phi ) }
\end{array} \quad \left\{\begin{array}{l}
\mathrm{ns}(\phi, 1)=\operatorname{coth}(\phi) \\
\operatorname{cs}(\phi, 1)=\operatorname{csch}(\phi) \\
\mathrm{ds}(\phi, 1)=\operatorname{csch}(\phi)
\end{array}\right.\right.
$$

for $k=0$ Eq. (43) contains Eq. (29) with trigonometric functions and $J_{z}$ and $J_{y}$ interchanged, and for $k=1$ it contains the same equation with hyperbolic functions and $J_{z}$ and $J_{x}$ interchanged.

The values of $J_{x}, J_{y}, J_{z}$ obtained from Eq. (43) for $\lambda=0$ and $\psi \neq 0$ corresponds to the trivial fixed point, which describes the non-interacting phase. Further on we consider only the case $r>0$. For $\psi=0$ the value $\lambda=0$ corresponds to one of the critical points, and $\lambda \in$ $(0,2 K(k))$ corresponds to the critical surface.

In Fig 4 we show the critical surface of one critical point. Due to the symmetry of equations mentioned in Sec. II, three other critical surfaces can be obtained from presented on Fig. 4 by rotations by the angle $\pi$ about the Cartesian axes. Fig. 5 with all four critical surfaces gives a complete picture of the phase diagram for the $X Y Z$ model, with critical surfaces separating between the infinite isotropic antiferromagnetic Heisenberg exchange phase and the phase, corresponding to the impurity spin decoupled from the electron environment.

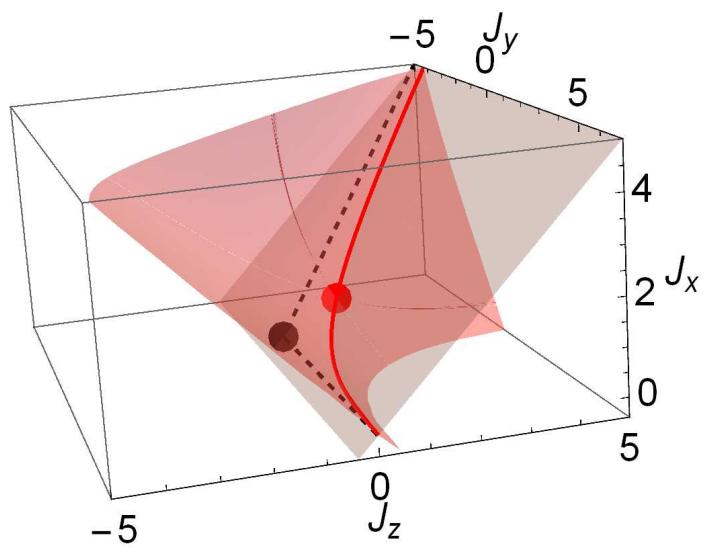

FIG. 4: (color online) Critical surface of the critical point $\left(J_{x}, J_{y}, J_{z}\right)=(1,1,1)$ is shown in red. Solid (dotted) line is a critical line (asymptotes) on $J_{x}=J_{z}$ plane (painted in grey); these lines are the same as in Fig. 1

As far as Kondo effect is concerned, we can repeat verbatim the last paragraph of Section $\amalg$, only this time Eq. (43) should be used instead of Eq. (29). 


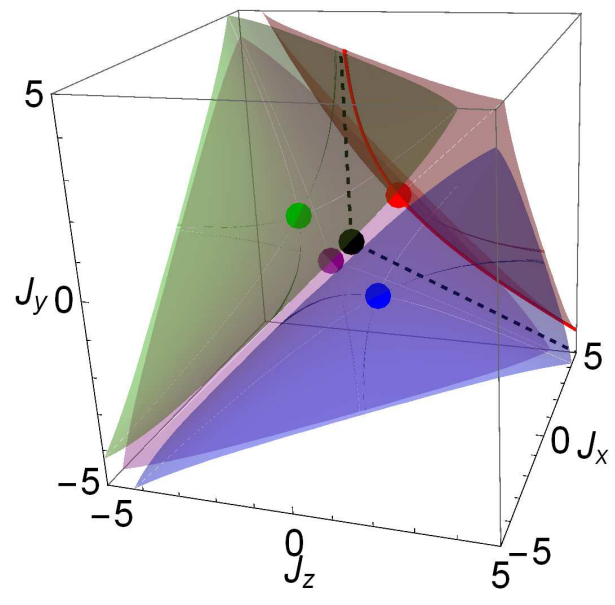

FIG. 5: (color online) All four critical surfaces. Critical points are denoted by colored circles.

\section{CONCLUSIONS}

We considered a single magnetic impurity described by the spin-anisotropic s-d(f) exchange (Kondo) model. We formulated the explicitly rotation invariant scaling equation for the power law electron DOS and solved this equation in terms of elliptic functions.

We found the infinite isotropic antiferromagnetic Heisenberg exchange phase, the phase, corresponding to the impurity spin decoupled from the electron environment (for the pseudogap DOS) and the infinite Ising exchange phase (for the DOS diverging at the Fermi level). We studied in details the critical surface corresponding to the finite isotropic antiferromagnetic Heisenberg exchange (for the pseudogap DOS).

\section{Acknowledgments}

This work has been supported in part by RIKEN iTHES Project and Molecular Systems. One of the authors (E.K.) thanks RIKEN for the hospitality extended to him during his stay. The other author (K.N.) is supported by Grant-in-Aid for JSPS Fellows (Grant No. 16J07637).

The authors are grateful to N. Andrei, Y. Avishay, K. Ingersent, D. Khveshchenko, T. Kimura, Chi-Cheng Lee, A. Mitchell, A. Nevidomskyy, Y. Ohyama, R. Sakano, Y. Teratani, and M. Vojta for valuable discussions, and particularly to $\mathrm{V}$. Yu. Irkhin for bringing to their attention Refs. 23 24 and to A. Oguri for bringing to their attention Refs. 31,33.

\section{Appendix A: If you have seen one, you have seen them all}

We explained in Section II] that if we know the quadratic terms in poor man's scaling equation for the Hamiltonian (4), we know these terms in poor man's scaling equation for the Hamiltonian (3). We claim that this remains true for the higher order terms. For example, from scaling equation for the isotropic Hamiltonian (44) ${ }^{2}$

$$
\frac{d J}{d \ln \Lambda}=-2 \rho J^{2}+2 \rho^{2} J^{3},
$$

follows scaling equation for reduced anisotropic Hamiltonian

$$
H_{e x c}=\sum_{i} J_{i} S^{i} s^{i}(0)
$$

in the form

$$
\begin{aligned}
& \frac{d J_{x}}{d \ln \Lambda}=-2 \rho J_{y} J_{z}+\rho^{2}\left(J_{y}^{2}+J_{z}^{2}\right) J_{x} \\
& \frac{d J_{y}}{d \ln \Lambda}=-2 \rho J_{x} J_{z}+\rho^{2}\left(J_{x}^{2}+J_{z}^{2}\right) J_{y} \\
& \frac{d J_{z}}{d \ln \Lambda}=-2 \rho J_{x} J_{y}+\rho^{2}\left(J_{x}^{2}+J_{y}^{2}\right) J_{z} .
\end{aligned}
$$

This result can be obtained by considering expression

$$
\sum_{i k l} J_{i} S^{i} \sigma^{i} J_{k} S^{k} \sigma^{k} J_{l} S^{l} \sigma^{l}
$$

and applying twice Eq. (9).

\section{Appendix B: Solving Eq. (32) using Jacobi elliptic functions}

For convenience of the reader we present here basic facts concerning Jacobi elliptic functions. There are three major functions: $\operatorname{sn}(\lambda, k)$ solves the differential equation

$$
\left(\frac{d u}{d \lambda}\right)^{2}=\left(1-u^{2}\right)\left(1-k^{2} u^{2}\right)
$$

$\operatorname{cn}(\lambda, k)$ solves the differential equation

$$
\left(\frac{d u}{d \lambda}\right)^{2}=\left(1-u^{2}\right)\left(1-k^{2}+k^{2} u^{2}\right)
$$

$\operatorname{dn}(\lambda, k)$ solves the differential equation

$$
\left(\frac{d u}{d \lambda}\right)^{2}=\left(1-u^{2}\right)\left(u^{2}-1+k^{2}\right) .
$$

Also, there are nine minor functions:

$$
\operatorname{pq}(\lambda, k)=\frac{\operatorname{pn}(\lambda, k)}{\mathrm{qn}(\lambda, k)},
$$


where $\mathrm{p}$ and $\mathrm{q}$ are any of the letters $\mathrm{n}, \mathrm{s}, \mathrm{c}, \mathrm{d}(\mathrm{nn}(\lambda) \equiv 1)$, named by the first letter of the numerator followed by the first letter of the denominator.

The rules of differentiation of the elliptic functions are:

$$
\begin{aligned}
& \left\{\begin{array} { l } 
{ \frac { d } { d \lambda } \mathrm { sn } = \mathrm { cn } \cdot \mathrm { dn } } \\
{ \frac { d } { d \lambda } \mathrm { cn } = - \mathrm { sn } \cdot \mathrm { dn } } \\
{ \frac { d } { d \lambda } \mathrm { dn } = - k ^ { 2 } \cdot \mathrm { sn } \cdot \mathrm { cn } }
\end{array} \quad \left\{\begin{array}{l}
\frac{d}{d \lambda} \mathrm{nc}=\mathrm{sc} \cdot \mathrm{dc} \\
\frac{d}{d \lambda} \mathrm{sc}=\mathrm{nc} \cdot \mathrm{dc} \\
\frac{d}{d \lambda} \mathrm{dc}=\left(1-k^{2}\right) \cdot \mathrm{nc} \cdot \mathrm{sc}
\end{array}\right.\right. \\
& \left\{\begin{array} { l } 
{ \frac { d } { d \lambda } \mathrm { ns } = - \mathrm { cs } \cdot \mathrm { ds } } \\
{ \frac { d } { d \lambda } \mathrm { cs } = - \mathrm { ns } \cdot \mathrm { ds } } \\
{ \frac { d } { d \lambda } \mathrm { ds } = - \mathrm { ns } \cdot \mathrm { cs } }
\end{array} \quad \left\{\begin{array}{l}
\frac{d}{d \lambda} \mathrm{nd}=k^{2} \cdot \mathrm{sd} \cdot \mathrm{cd} \\
\frac{d}{d \lambda} \mathrm{sd}=\mathrm{cd} \cdot \mathrm{nd} \\
\frac{d}{d \lambda} \mathrm{cd}=-\left(1-k^{2}\right) \cdot \mathrm{sd} \cdot \mathrm{nd}
\end{array}\right.\right.
\end{aligned}
$$

(the argument of all functions is $\lambda$, and the modulus is $k)$.

These simple rules allow to integrate Eq. (32) just by inspection. In fact, there are four options for the signs of $P, Q, R$ in Eq. (32): three pluses, two pluses and one minus, one plus and two minuses, and three minuses. Equation (B5) allows one to solve Eq. (32) for each option; one should just read two previous paragraphs backward. Thus Eq. (21) is solved by the set of functions (in the domain $\widetilde{J}_{x}^{2}>\widetilde{J}_{z}^{2}>\widetilde{J}_{y}^{2}$ )

$$
\begin{aligned}
& \widetilde{J}_{x}=\operatorname{ns}(\lambda, k) \\
& \widetilde{J}_{y}=\operatorname{cs}(\lambda, k) \\
& \widetilde{J}_{z}=\operatorname{ds}(\lambda, k) .
\end{aligned}
$$

Action on this solution by the group of transformations $\lambda \rightarrow \lambda+\psi$ and $\lambda \rightarrow A \lambda, \widetilde{J} \rightarrow \widetilde{J} / A$ gives the 3 -parameter family of solutions 31,33

$$
\begin{aligned}
& \widetilde{J}_{x}=A \cdot \operatorname{ns}(A \lambda+\psi, k) \\
& \widetilde{J}_{y}=A \cdot \operatorname{cs}(A \lambda+\psi, k) \\
& \widetilde{J}_{z}=A \cdot \operatorname{ds}(A \lambda+\psi, k),
\end{aligned}
$$

wherefrom follows Eq. (43). Permutation of the indices $x, y, z$ gives solutions in other domains.

\section{Appendix C: Let a hundred flowers blossom}

The two representations of the solution we got (through Weierstrass and through Jacobi elliptic functions) in spite of looking differently, are equivalent. Weierstrass elliptic function can be expressed through Jacobi one using equation

$$
\mathcal{P}(\lambda)=e_{3}+\frac{e_{1}-e_{3}}{\mathrm{sn}^{2} w},
$$

where $e_{1,2,3}$ are three roots of the RHS of Eq. (39), considered as a polynomial, and where the modulus $k$ of the Jacobi function equals

$$
k \equiv \sqrt{\frac{e_{2}-e_{3}}{e_{1}-e_{3}}}
$$

and it's argument $w$ equals

$$
w \equiv z \sqrt{e_{1}-e_{3}} .
$$

Starting from Eq. (32) we get

$$
e_{1}=-R S y_{0}, \quad e_{2}=-Q S z_{0}, \quad e_{3}=-Q R x_{0} .
$$

Thus for $Q=R=S=-1$ we recover Eq. (B7).

\section{Appendix D: Special elliptic cones}

To understand the geometric meaning of the solution (43) let us start from elementary geometry. The Euclidean space $(x, y, z)$ can be in a unique way foliated into the elliptic cones of special $(\alpha+\beta+\gamma=0)$ type

$$
\alpha x^{2}+\beta y^{2}+\gamma z^{2}=0 .
$$

(For $\alpha=0$ or $\beta=0$ the special cone is a pair of planes.)

The foliation includes three families of cones (the axis of the cones of each family is one of the Cartesian axes). These families will be referred to as $x$-cones, $y$-cones and $z$-cones. An apex angle of a given cone is the angle between the cone's axis and the section of the cone by a plane which contains the cone axis. For example, for a $z$-cone, $\theta_{z x}$ is the angle between the $\mathrm{OZ}$ axis and the section of the cone by the plane $x=0$. It is obvious that

$$
\cos \theta_{z x}=-\frac{\beta}{\gamma} .
$$

Now let us go from geometry to calculus. Recalling the identity, elliptic functions satisfy

$$
1-k^{2}+k^{2} \operatorname{cn}^{2}(\lambda, k)-\operatorname{dn}^{2}(\lambda, k)=0,
$$

one realizes that the solution (43) satisfies

$$
\left(1-k^{2}\right) J_{x}^{2}+k^{2} J_{y}-J_{z}^{2}=0 .
$$

Hence the special cones in the phase space $J_{x}, J_{y}, J_{z}$ remain invariant under the evolution (this is why they were introduced above), and the parameter $k^{2}$ in the solution (43) is the cosine of the $\theta_{z x}$ apex angle of the special cone, the solution belongs to. Note that the stable fixed point $\left(J_{x}, J_{y}, J_{z}\right)=(0,0,0)$ of Eq. (15) is the apex of all special cones ${ }^{34}$.

\section{Appendix E: Mathematical conclusions}

In this paper we have integrated specific systems of $m$ coupled scaling equations in terms of known functions. Two specific features of the systems allowed us to do it. First, it was possible to reduce each system to the form

$$
\frac{d \widetilde{J}_{n}}{d \lambda}=R_{n} \frac{\Phi(\widetilde{J})}{\widetilde{J}_{n}}
$$


where $\Phi$ is some function of the coupling constants, and $R_{n}$ are constants. This allowed us to obtain $m-1$ first integrals of the system. Geometrically this means that in the space with the coordinates $\left(x_{1}, \ldots x_{m}\right)=\left(\widetilde{J}_{1}^{2} \ldots \widetilde{J}_{m}^{2}\right)$ a flow line of Eq. (E1) lies on an arbitrary ray in the direction $\left(R_{1}, \ldots R_{m}\right)$.

The integrals being found, we are left with a single differential equation for a single variable (whatever function of the coupling constants is chosen as such variable). This equation is of the first order, solved with respect to the derivative of the dependent variable, and has the RHS which does not contain the independent variable. It means that a system which can be reduced to Eq. (E1) (for any $\Phi$, any $m$ and any set of constants $R_{n}$ ) can be integrated in quadratures.

Second, the function $\Phi$ being what it was, we were able to write down the solutions in terms of known transcendental functions (circular trigonometric and hyperbolic for $m=2$ and elliptic for $m=3$ ). Jacobi elliptic functions appear when we chose a coupling constant (or inverse coupling constant, or the ratio of two coupling constants, as we understand looking at Eq. (B5)) as the above mentioned variable. Weierstrass elliptic functions appear when the square of a coupling constant is chosen.
* Electronic address: Eugene.Kogan@biu.ac.il

1 P. W. Anderson, J. Phys. C 3, 2439 (1970).

2 A. C. Hewson, The Kondo Problem to Heavy Fermions, (Cambridge University Press, Cambridge, 1993).

3 K. Sengupta and G. Baskaran, Phys. Rev. B 77, 045417 (2008).

4 T. O.Wehling, A. V. Balatsky, M. I. Katsnelson, A. I. Lichtenstein, and A. Rosch, Phys. Rev. B 81, 115427 (2010).

5 M. Vojta, L. Fritz, and R. Bulla, Europhys. Lett. 90, 27006 (2010).

6 B. Uchoa, T. G. Rappoport, and A. H. Castro Neto, Phys. Rev. Lett. 106, 016801 (2011); 106, 159901(E) (2011).

7 L. Fritz and M. Vojta, Rep. Prog. Phys. 76, 032501 (2013).

8 J.-H. Chen, L. Li, W. G. Cullen, E. D. Williams, and M. S. Fuhrer, Nat Phys 7, 535 (2011).

9 M. Vojta and R. Bulla, Eur. Phys. J. B 28, 283 (2002).

10 A. K. Zhuravlev, V. Yu. Irkhin, Phys. Rev. B 84, 245111 (2011)

11 T. Kanao, H. Matsuura, and M. Ogata, J. Phys. Soc. Jpn. 81, 063709 (2012).

12 M. A. Cazalilla, A. Iucci, F. Guinea, and A. H. Castro Neto, arXiv: cond-mat/1207.3135.

13 A. K. Mitchell and L. Fritz, Phys. Rev. B 88, 075104 (2013).

14 A. K. Mitchell, M. Vojta, R. Bulla, and L. Fritz, Phys. Rev. B 88, 195119 (2013).

15 T. Shirakawa and S. Yunoki, Phys. Rev. B 90, 195109 (2014); ibid 93, 205124 (2016).

16 D. Withoff and E. Fradkin, Phys. Rev. Lett. 64, 1835 (1990).

17 K. Chen and C. Jayaprakash, J. Phys.: Condens. Matter 7, L491 (1995).

18 C. Gonzalez-Buxton and K. Ingersent, Phys. Rev. B 57, 14254 (1998)

19 R. Bulla, Th. Pruschke, and A. C. Hewson, J. Phys.: Condens. Matter 9, 10463 (1997).

20 R. Bulla, M. T. Glossop, D. E. Logan, and Th. Pruschke, Journal of Physics: Condensed Matter 124899 (2000).
${ }^{21}$ M. T. Glossop and D. E. Logan, Eur. Phys. J. B 13, 513 (2000).

22 L. Fritz and Matthias Vojta, Phys. Rev. B 70, 214427 (2004).

23 D.L. Cox and A. Zawadowski, Adv. Phys. 47, 599 (1998).

24 V. Yu. Irkhin, M.I. Katsnelson and A.V. Trefilov, Zh. Eksp. Teor. Fiz. 105, 1733 (1994); Physics Letters A 213, 65 (1996).

25 Ph. Nozieres and A. Blandin, J. de Phys. 41, 193 (1980).

${ }^{26}$ L. D. Landau and E. M. Lifshitz, Landau and Lifshitz Course of Theoretical Physics: Vol. 3 Quantum Mechanics, (Pergamon Press, 1991).

27 L. D. Landau, A. A. Abrikosov, and I. M. Khalatnikov, Dokl. Akad. Nauk SSSR 95, 497 (1954).

28 A. Z. Patashinskii and V. L. Pokrovskii, Fluctuation Theory of Phase transitions, 2 ed., (Moskow. Nauka, 1982) [in Russian]; English translation of the 1 ed see: Fluctuation Theory of Phase transitions, (Pergamon Press, 1979)

29 M. Cheng, T. Chowdhury, A. Mohammed, and K. Ingersent, preprint arXiv:1702.06515

30 L. D. Landau and E. M. Lifshitz, Landau and Lifshitz Course of Theoretical Physics: Vol. 1 Mechanics, (Elsevier, 1976).

31 H. Shiba, Prog. Theor. Phys. 43, 601 (1970).

32 M. Abramowitz, I. A. Stegun eds., Handbook of Mathematical Functions with Formulas, Graphs, and Mathematical Tables, (National Bureau of Standards, Washington, 1964).

33 K. Yosida, Theory of Magnetism (Springer, Berlin Heidelberg New York, 1996).

34 I. A. Brodsky,

And Euclid did not know that approaching the apex of the cone,

A thing is going to meet not it's end, but eternity.

-I sit by the window (1964) 